\title{
The Research on Mass-MAS Parallel Decision in Integrated Infrastructure for Agile Enterprise
}

\author{
J.L. Su* \\ TSL School of Business and Information Technology, Quanzhou Normal University, 362000, Quanzhou, P. R. China
}

\begin{abstract}
The Information Economics and its platform can input the vigor into manufacturing industry, i.e. garment industry, and help to increase its ability for good return, can promote the level of technology and management of Garments from a labor-intensive industry, and can move it toward the accurate management. The platform is designed as Comprehensive Information Platform by our research team. This paper is one of the series of papers about the design of Agile Infrastructure for Collaborative Manufacturing as well as Agile Supply Chain. This one lays emphasis upon the design of the optimal production planning algorithm to keep the manufacture financing stability in product cycle. We mainly concern and research into the research on Mass-MAS parallel decision in integrated infrastructure for agile enterprise, including the analysis of information exchange rules for MAS members, individual behavior rules and its correction by environment driven, MAS decision rules for members in the Algorithm, the solution to the problem \& algorithm design in the decision-making operation burden for IIAE. Their logical relationship and flow chart designs are elaborated in turn. The paper tries to introduce the research of Agile Infrastructure and its key technologies, showing how well they work in some traditional manual industries.
\end{abstract}

Keywords: IIAE, manufacturing industry, mass-MAS parallel decision.

\section{INTRODUCTION}

With the growing trend of economic and informationbased globalization, manufacturing industry has to face the increasingly fierce competition as well as the frequent and unpredictable market fluctuations. An Agile theory thereupon emerges as the requirement of global market fluctuations. The life cycles of products are shortened and the pace of the product renewal is quickened, and the demands of customers are becoming more and more specific and diversified, thereupon, the producing and organizing models transfer from product-oriented to customer-oriented, requirementoriented and service-oriented, and the aims of enterprises transfer from enterprise profit-driven to market and social profit-driven. The key for enterprise to gain its markets and customers is to improve some factors, such as time, quality, cost, service and environment. Agile Enterprise and Manufacturing Enterprise Alliance, as the running models of future enterprises, will fully make use of new technologies and coordinated operation which is more agile, compartmentalized, order-driven and dynamic to adapt to the markets [1].

Integrated Infrastructure for Agile Enterprise (IIAE) is an operating platform of some new operating conceptions of enterprises, such as Agile Manufacturing Enterprise (AME) and Agile Supply Chain (ASC). In this thesis, the research on the IIAE is not done traditionally, because it reforms the enterprises to be information-based and agile from outside, and lays down criteria for the platform. Driven by order and profit, the enterprises transfer to be Agile Enterprises (AE) after joining in the platform and reform abiding by the norms of output and input of platform operations. The IIAE provides paid service for Agile Enterprise, and promotes the advanced management model which is standard, effective and information-based in production, sales, policy-making, financial affairs, personnel affairs and storage. It also provides services of order cooperation, sales cooperation, material supply cooperation, post-sale service cooperation, storage and transportation cooperation, capital cooperation, market analysis and policy-making. The member enterprises will be devoted respectively to what they are expert in [2]. Consequently, the ASC with dynamic members is established. The members of ASC negotiate abiding by common rules and accomplish the tasks of production and sale from the outside market, which is Cooperative Games. In the IIAE, the design of construction and its model, the managing policy of supply chain, the agile manufacturing technology, communication and information processing, are the key problems demanding prompt solutions.

The relationship among Agile Manufacturing Enterprise, Agile Supply Chain and Agile Infrastructure is like the relation between the sharpness of knife and the knife itself, which cannot be divided apart. Agile Infrastructure for Manufacturing System is the platform where the Agile Enterprise, Agile Supply Chain, Agile Manufacturer, Virtual Enterprise are put to good use. The essential condition of Agile Enterprise and Virtual Enterprise is the Agile Infrastructure which is reliable, cross-enterprise, cross-industry and transregional. The Agile Infrastructure is established to normalize the managerial practices of enterprise, such as production, sale, policy-making, financial affairs and personnel affairs. The member enterprises can be inserted flexibly, just like the circuit module with standard output and input jacks. In the 
Agile Infrastructure, member enterprises run business with common rules and establish dynamic Agile Enterprise Alliance, i.e. Virtual Enterprise. The members of Agile Enterprise Alliance negotiate abiding by common rules and accomplish the task of production and sale, which is a game of cooperation. According to the outside market environment and the group intention of inside members, the Virtual Enterprises make identical judgment and macrocosmic layout [3-5].

As to the problems in the construction of IIAE, the author mainly researches on some issues, such as the IIAE and its model, the managing technology of the ASC in the IIAE, the agile manufacturing technology based on Network, the communication strategy in IIAE and its image information processing. These issues respectively belong to the agile model construction, agile managing technology, agile manufacturing technology, and agile informational technology (i.e. communication technology and image information processing). These matters are under thorough and meticulous consideration in this series of papers.

In this paper, the authors research into the construction of Agile Infrastructure for Collaborative Manufacturing and Agile Supply Chain and its key enabled technologies. It is supported by the achievements of some projects, such as the "Decision-making Research on Intelligent Manufacture Equipment Cluster" (2014J132) which is a Science and technology project of Quanzhou City.

This paper, which lays emphasis upon the optimal production planning includes the problem in manufacture financing by optimal product, the analyzed of chaotic neural network optimization algorithm, the solution \& algorithm deign in optimal strategy for produce, the isometric increase example, the nonisometric increase example, and the example with unsymmetrical matrix. The overall organization of the paper is as follows. After the introduction, in Section II we present the Agile Infrastructure and it's application. In section III, the problem in the decision-making operation burden for IIAE, are touched upon. Then, the analyzed of Information Exchange Rules for MAS Members is presented on Section IV. In Section V VI, the authors elaborate the problem's solution \& programming in MAS decision rules for members in the algorithm. After that, Individual Behavior Rules and Its Correction by Environment Driven are introduced in Section VI. Finally, Section VII concludes the paper.

\section{AGILE INFRASTRUCTURE AND IT'S APPLICA- TION}

Agile Infrastructure for Collaborative Manufacturing and Agile Supply Chain can standardize the output and input information of its member enterprises. As can be seen from Fig. (1), Our team had done a lot on Agile Infrastructure research; there is the Detail of Structure of IIAE. IIAE stores the manufacturing and supply chain and human resource information in it's database and helps the enterprises to run their business in an Agile way designed by IIAE, i.e. Agile Collaborative Manufacturing Execution Systems (ACMES), Enterprise Resource Planning (ERP), Customer Relationship Management (CRM), Product Data Management (PDM), Supply Chain Management (SCM) [6].

The Comprehensive Information Platform tries to affect every aspect of its members in their running mechanisms, and each registered enterprise becomes a standardized module of the Agile Infrastructure. All its members can build up Agile Manufacturing Enterprise, or construct Agile Supply Chain temporarily or permanently. Thus the Enterprises can concentrate on their core competences and they are able to recombine rapidly their interior and exterior capabilities and resources, thereby to respond rapidly to the market opportunity.

\section{PROBLEM: THE DECISION-MAKING OPERA- TION BURDEN FOR IIAE}

Study on the integrated infrastructure for agile enterprises in the manufacturing industry, we have constructed a central platform, which can accomplish the communication and control of all kinds, but agile enterprise members in the platform

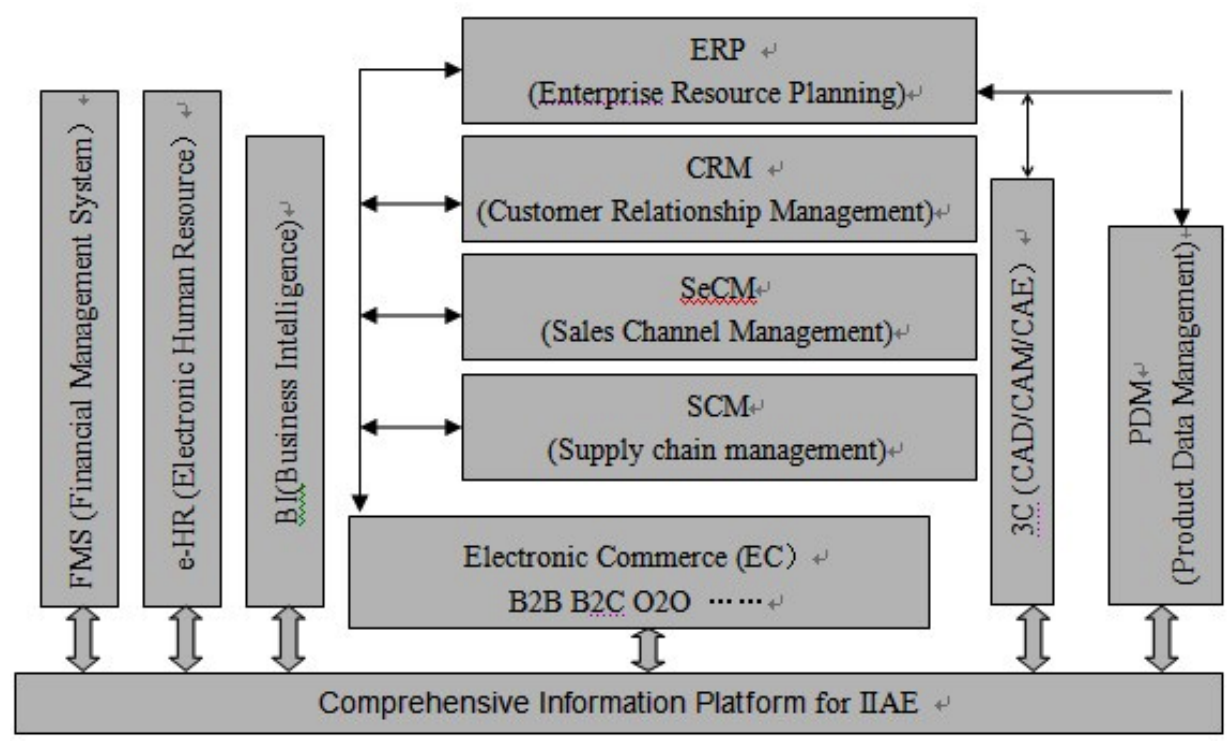

Fig. (1). The detail of structure for IIAE. 
under the framework of decision still needs further research. We used to tend to make unified decision by the central computers of the platform, but with the number of members of the more complex, the central platform decision increasingly heavy burden, the amount of communication between individual members are growing exponentially [7]. Therefore, we have to re investigate the agile enterprise integrated structure decision method. We have made a part of the theory and simulation experiment results for Mass-MAS parallel decision algorithm, but everything is still just beginning......

\section{ANALYSE: INFORMATION EXCHANGE RULES FOR MAS MEMBERS}

Parallel decision communication platform itself is not involved in decision making, it is simply the agent communication platform, the same with the air in the cave, who help the ant chemical signal transmission. At the same time, input and output interface of MAS communication platform is the whole system of exchanging information with the outside world. Agent does not communicate directly, all decisions by voting out of the way, between the individual and the individual competition and game also goes through the decisionmaking platform. For example, a damaged agent will quickly be replaced with normal agent function.

We design all agents are first to enthusiastically for the tasks. And there are no desires and no fears, the agents are not to hesitate to anything in order to finish the task. Decision platform like a job list to offer the job, and the individual members in the first task registered its competitive position, get the chance to work.

The platform can be wired communication or wireless communication. Decision and game respectively study isomorphism of MAS and heterogeneous MAS.

The current MAS system, wired or wireless network, communication local area network topology structure mainly includes two types: no center call for equality (Peer to Peer) topology, with a center (Hub-Based) topology. No center network topology requirements for any of two Agents can be direct communication in the network, the network using public broadcast communication, the Agent using the CSMA type multiple access protocol of competition in public channel. The advantage of the structure is the network survivability, convenient networking, and low cost. But when the network Agent number too much, channel competition has become the bottleneck of limiting the performance of the network. And for the mobile Agent, to meet the two Agent direct communications, network Agent layout will be subject to environmental constraints [8-11]. So, this topology suitable working group on Agent network scales fewer.

Topology with center requires an Agent acts as a central station, access to all Agent on the network are by the control. So, when the network traffic increases, the throughput and delay performance of network would not drastic deterioration. For mobile Agent, each Agent needs only in the coverage area of the Centre-Agent, and then it can communicate with the others. So the network Agent layout being less restrictive by the environment, and it has stronger ability to adapt to the environment. Center network topology has its fatal weakness, that is his overall communication and data throughput by center Agent limitations [12]. The other prob- lem is the invulnerability ability of the center network topology, because the fault of Centre-Agent will easily lead to paralysis of the entire network.

Platform communication mode is different from any existing methods, it provides a more powerful communication center station, the central station is the professional data transmission and storage centers, with data throughput capacity, strong high reliability, high security, support for multi Agent and communication. Information platform does not participate in the decision task, do not consider the completion of the task, it makes all Agent members understand the global information; it provide decision-making basis and consultative platform for all the members. On the platform of data throughput capacity range, the system does not limit the number of Agent. Individual members in the MAS access to information is the main way of broadcasting mode, but it can also be query mode [13]. Although no communication between individuals, but can be made by the query to other individual information platform. Individual members have the ability to feel the outside environment, can be their own access to the information and its activity status, and constantly or periodically in transmission and storage of data to the platform [14].

\section{MAS DECISION RULES FOR MEMBERS IN THE ALGORITHM}

In the robot group, robot according to certain strategy, coordination mechanism, the control laws of organic and organized, the robot through the organizational structure of knowledge to obtain a global view on the overall system behavior, so as to take effective strategies to guide the local control to achieve collaboration. Multi robot coordination refers to multiple robots with different target of reasonable arrangement of its objectives, resources, to adjust their own behavior, cooperate to achieve a common goal. We believe that cooperation is a special type of competition. Vote on the MAS member is a game. Agents don't know each other contents of the voting, independent with the last game, their condition and other information known to the whole system, to make a favorable judgment $[15,16]$.

Different voting decision scheme is likely to get the votes be well-matched in strength, so the decision result is not clear, at this time should be done second times the voting decision, and the whole process can be viewed as a complex, cooperative game [17]. In considering the parallel decision problem of MAS system, we study Nash and his followers in the non cooperation game and the cooperation achievements.

On the basis of consistency is the discussion of each decision maker on the corresponding weight vector criterion according to the method of AHP.

$$
w^{d}=\left(w_{1}^{d}, w_{2}^{d}, \cdots, w_{n}^{d}\right)
$$

And the opinions of decision makers is consistent, it is necessary to research the problem of comparability of weighted vector

$$
w^{d}(d=1,2, \mathrm{~L}, m) \text {. }
$$

W. Cook and M. Kress proposed by L1 space model method [8]: L. Hamer proposed cosine law two to clamping angle; 
$\mathrm{N}$. Bryson is proposed to measure the similarity weighted vector with

$$
S\left(w^{d}, w^{r}\right)=1-\sin \left(w^{d}, w^{r}\right)
$$

Method of N. proposed by Bryson has intuitive geometric meaning and in the application are also more convenient, but because in the actual problem, decision makers on each of the two criteria of the more general may not be an exact real numbers $a_{i j}$.

And expressed by "vague language between $a_{i j}$ left or right", that is $\left(c_{i j}, a_{i j}, b_{i j}\right)$, a triangular fuzzy number to represent it more realistic.

Hsu presented the concept of using trapezoidal fuzzy numbers, the conversion problem of similarity enables decision makers to given criteria evaluation and scheme of the trapezoidal two area ratio.

3.1 Similarity metric function and coincidence indicator

Let

$$
\begin{aligned}
& w_{1}=\left(l_{1}, m_{1}, u_{1}\right), \\
& w_{2}=\left(l_{2}, m_{2}, u_{2}\right)
\end{aligned}
$$

of two triangular fuzzy numbers, $u_{1}(x), u_{2}(x)$ the corresponding membership grade, then said :

$$
\begin{aligned}
& S\left(w_{1}, w_{2}\right)=\int_{x} \min \left(u_{1}(x), u_{2}(x)\right) d x \\
& / \int_{x} \max \left(u_{1}(x), u_{2}(x)\right) d x
\end{aligned}
$$
paste.

As a function of the number of $w_{1}, w_{2}$ similarity triangle

$$
\text { Let } w^{d}=\left(w_{1}^{d}, w_{2}^{d}, \cdots, w_{n}^{d}\right), w^{r}=\left(w_{1}^{r}, w_{2}^{r}, \mathrm{~L} w_{n}^{r}\right)
$$

be two individual triangular fuzzy vectors, and then

$$
S\left(w^{d}, w^{r}\right)=\left(\begin{array}{l}
S_{1}\left(w_{1}^{d}, w_{1}^{r}\right), \\
S_{2}\left(w_{2}^{d}, w_{2}^{r}\right), \\
\mathrm{L}, \\
S_{n}\left(w_{n}^{d}, w_{n}^{r}\right)
\end{array}\right)
$$

represent the similarity metric vectors of $w^{d}$ and $w^{r}$.

Let $w^{G}, \quad w^{d}(d=1,2, \mathrm{~L}, m)$ as triangular fuzzy vector, then

$$
\left\{\begin{array}{l}
G I=\sum_{d=1}^{m} S\left(w^{G}, w^{d}\right) / m \\
I I=\sum_{d=1}^{m} S\left(w^{G}, w^{d}\right) / m
\end{array}\right.
$$

$G I$ represents the group consistency vector index about $w^{G}$, as well as $I I$ represents the individual identity of vector index about $w^{r}$.
We assume that the each component of $G I$ are

$\sum_{d=1}^{m} S_{i}\left(w_{i}^{G}, w_{i}^{d}\right) / m \geq \alpha,(i=1,2, \mathrm{~L}, n)$,

then the group is consistency in level $\alpha$ about $w^{G}$. If there exists a integer $1 \leq j \leq n$, then

$$
\sum_{d=1}^{m} S_{i}\left(w^{G}, w_{i}^{d}\right) / m<\alpha,(i=1,2, \cdots, n)
$$

And we can see the group is not consistency in level $\alpha$ about $w^{G}$.

In the above expression, $0<\alpha<1$, according to the actual problem level $\alpha$ is given by the group voting or group chairman. To maintain the consistency of the whole group requires groups on each criterion are the same.

3.2 The methods for searching group consistency

Let $I_{i}^{d}=\sum_{r=1}^{m} S_{i}\left(w_{i}^{d}, w_{i}^{r}\right) / m$ represents the coincidence indicator which relative NO. $i$ rule by the decision maker $d$.

Let $I_{i}^{d}(d=1,2, \mathrm{~L}, m)$ by arrive greatly small ordinal. The larger the number $I_{i}^{d}$, the more $w_{i}^{d}$, the corresponding weighted vector of $d$, can represent the majority of the wishes of the people. Or in a group, the relative NO. $i$ rule weights more.

$$
w_{i}^{G}=\left(\sum_{d=1}^{m} I_{i}^{d} w_{i}^{d}\right) / \sum_{i=1}^{m} I_{i}^{d},
$$

$(i=1,2, \cdots, n)$

is group decision weighted component. Thus the weighted vector for group decision making can be $w_{d}^{G}=\left(w_{1}^{G}, w_{2}^{G}, \mathrm{~L}, w_{n}^{G}\right)$. And for any number of $r, d$, if $w_{i}^{d}=w_{i}^{r}$ there is

$$
w^{G}=\left(w_{1}^{G}, w_{2}^{G}, \mathrm{~L}, w_{n}^{G}\right)
$$

Let $w_{i}^{G}, w_{i}^{d}(d=1,2, \mathrm{~L}, m)$ be the triangular fuzzy vector, and then $\prod_{d=1}^{m} w_{i}^{d} \subseteq w_{i}^{G}$. So,

$\left(\prod_{d=1}^{m} w_{1}^{d}, \prod_{d=1}^{m} w_{2}^{d}, \mathrm{~L}, \prod_{d=1}^{m} w_{n}^{d}\right) \subseteq w^{G}$

Test vector $w^{G}$, to see whether it can make the group consensus. If it is, then $w^{G}$ is the final weight vector of group decision making. Otherwise,

$s_{i}^{d}=I_{i}^{d} / I_{i}^{d(T M)},(d=1,2, \mathrm{~L} m), I_{i}^{d(T M)}$

is the average values of all the component vectors after $I_{i}^{d}$ in vector group $I_{i}$.

Let 


$$
S=\max _{d}\left\{S_{i}^{d}\right\}=\max _{d}\left\{I_{i}^{d} / I_{i}^{d(T M)}\right\},
$$

and because the finiteness of the number of $s_{i}^{d}$, there must be $m_{i}$ who let $s=s_{i}^{m_{i}}$. According to the structure of $S$, we can know that there could well be a big gap between $I_{i}^{m_{i}}$ and $I_{i}^{d(T M)}$, which represent the average values of all the component vectors after $I_{i}^{m_{i}}$.

Because each sub vector of $I_{i}$ is arranged from big to small, then the corresponding decision makers of $I_{i}^{1}, I_{i}^{2}, \mathrm{~L} I_{i}^{m_{i}}$ will represent the majority of the wishes.

If $m_{i}=m$ then there are no significant differences among

$$
I_{i}^{1}, I_{i}^{2}, \mathrm{~L} I_{i}^{m_{i}}, \text { and } w_{i}^{G}=\left(\sum_{d=1}^{m} w_{i}^{d}\right) / m
$$

is final vector group decision.

$$
\text { If } m_{i}<m \text { then } w_{i}^{G}=\left(\sum_{d=1}^{m} w_{i}^{d}\right) / m \text { is the average decision }
$$

vector of all decision makers before $m_{i}$.

To examine each criterion $R_{i}(i=1,2, \mathrm{~L}, n)$ with the above method, then

$$
w^{G}=\left(w_{1}^{G}, w_{2}^{G}, \mathrm{~L}, w_{n}^{G}\right)
$$
then

Let $w_{i}^{G}, w_{i}^{G} \quad d=1,2, \mathrm{~L}, n$ be triangular fuzzy vector,

$$
\bigcap_{d=1}^{l_{i}} w_{i}^{d} \subseteq w_{i}^{G}
$$

So

$$
\begin{aligned}
& \left(\prod_{d=1}^{l_{i}} w_{1}^{d}, \prod_{d=1}^{l_{i}} w_{2}^{d}, \mathrm{~L}, \prod_{d=1}^{l_{i}} w_{n}^{d}\right) \subseteq w^{G} \\
& \left(l_{i} \text { is } m_{i} \text { or } m\right)
\end{aligned}
$$

The method according to the size of the individual vectors to determine how much the group consistency information of individual contains, in order to seek a group weighted vector, relative size from the whole inspects each individual vectors containing group consistency information, through the screening method to find the will of individual decision-making plays a key role in the decision-making process of this group, to structure of population weighted vector.

Although, Nash often emphasize the alliance member's aggressive and selfish problem and obtain the maximum interests in its elaboration on the ultimate goal; and we will define the agent member is fully selfless, cooperation, considering their interests as a whole, to obtain the maximum benefit for the ultimate goal; but because each member agent in different environment to experience different content, the resulting decision is different, and they are trying to stick in the game more inclined to the original policy, so the generated for a supply of sth..
Therefore, we believe that in this process, the research achievements of Nash and many of subsequent researchers are still applicable, it is due to these differences, which makes the whole cooperative game process needs to make some corresponding correction. At the same time, we may need to expand the cooperation game some theory and its application.

\section{INDIVIDUAL BEHAVIOR RULES AND ITS COR- RECTION BY ENVIRONMENT DRIVEN}

In fact is the symbol reasoning into individual MAS, which formed a kind of independent rules based on experience and environment correction, and thereby affect the whole system decision results, and thus improve the whole MAS long-term planning ability. Large groups of parallel decision mechanism is a kind of follow the "environmental, behavior, reaction" from the bottom to the parallel decision algorithm. All the algorithms are design for large groups of parallel decision mechanism of decision-making.

The main difference between our algorithm and "hierarchical" algorithm \& "hybrid" algorithm is the bottom-up decision structure of non hierarchical management; common features are symbolic reasoning rules based on individual behavior correction system using. Our algorithm for large groups of parallel decision mechanism of decision-making and "inclusive" algorithm has common points. That is their main characteristics of each subsystem of parallel work, and has the same "based on environmental behavior response" mode of intelligent system construction. And the difference of them lies in the large group of parallel decision mechanism decision making for a vote on the communication platform using the game theory, but not making decisions by communications within the group members. And secondly, large groups of parallel decision mechanism member individual behavior rule is context driven, has its own rules of symbolic reasoning based on the correction ability, follow the organisms return mode [18].

The members behavior system of our algorithm has define members' behavior rules, according to changes in the environment of independent study ability. The algorithm packaging Rules of conduct should have control of robot perception, exploring, obstacle avoidance, planning and task execution ability [19]. The behaviors are composed of a sensor to a local drive mapping, and the implementation of global target for the system through interaction behavior, so it has strong real-time performance.

In this case we consider the use of symbolic reasoning in the compensatory fuzzy neural network to simulate the way of the agent to obtain the rule revision pattern of return from the environment and experience.

Fuzzy core neural networks have emerged as an important tool for classification, which includes the Bayesian classification theory, the role of posterior probability in classification, posterior probability estimation via neural networks, and the relationships between neural networks and the conventional classifiers. As we know, the neuralnetwork-based control of the dynamic systems compensates the effects of nonlinearities and system uncertainties, so the stability, convergence and robustness of the control system 
can be improved. The fuzzy neural network possesses the advantages of both fuzzy systems and neural networks. Recent research activities in fuzzy core neural classification have established that FNN are a promising alternative to various conventional classification methods [20].

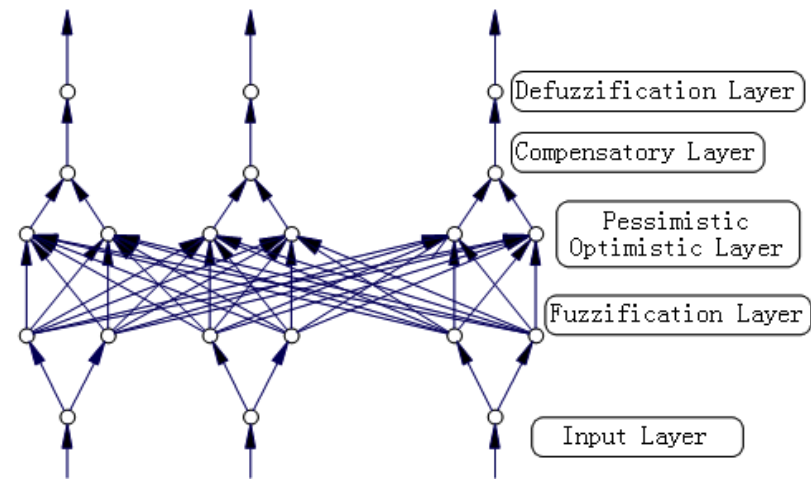

Fig. (2). Structure of CFNN.

This paper describes a fuzzy optimization model we have developed, which allows us to control the compression rate of sequences of images derived from Multi-sensors-fusion, and to relieve the congestion in GPRS communication. The model is a fuzzy core neural network (FNN); see Fig. (2).

The fuzzification procedure maps the crisp input values to the linguistic fuzzy terms with the membership values between 0 and 1.The Rule Base stores the rules governing the input and output relationship for fuzzy controller. The inference mechanism is responsible for decision making in the control system using approximate reasoning. The operations involved in this study are "AND". For CFNN, the inference mechanism includes pessimistic-optimistic operation layer and compensatory operation layer. The pessimisticoptimistic operation layer includes two types of cells: pessimistic compensatory cells and optimistic compensatory cells. Pessimistic compensatory cells always come up with a solution for the worst situation, while optimistic compensatory cells always choose a solution for the best situation. The compensatory fuzzy neuron can map the pessimistic input as well as the optimistic input to the compensatory output, which may lead to a relatively compromised decision for the situation between the worse case and the best case. The defuzzification procedure maps the fuzzy output from the inference mechanism to a crisp signal [21].

As we known by [6], a neurofuzzy system is a model with linguistic IF-THEN rules. The $m$ fuzzy IF-THEN rules

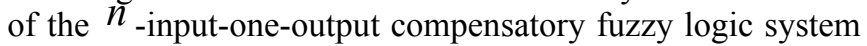
which used in compression system, can be defined as:

FR(k): IF $x_{1}$ is $A_{1}^{k}$ and... $x_{i}$ is $A_{i}^{k}$ and... $x_{n}$ is $A_{n}^{k}$ THEN $y$ is $B^{k}$

Where $A_{i}^{k}$ and $B^{k}$ are fuzzy membership functions, which are defined as:

$\mu_{A_{i}^{k}}\left(x_{i}^{p}\right)=\exp \left[-\left(\frac{x_{i}^{P}-a_{i}^{k}}{\sigma_{i}^{k}}\right)^{2}\right]$
$\mu_{B^{k}}\left(y^{p}\right)=\exp \left[-\left(\frac{y^{p}-b^{k}}{\delta^{k}}\right)^{2}\right]$

For $i=1,2, \mathrm{~L}, n$ and $k=1,2, \mathrm{~L}, m$.

With a compensatory degree $\gamma \in[0,1]$, the output of compensatory fuzzy neural network may has the following representation:

$$
f(x)=\frac{\sum_{k=1}^{m} b^{k} \delta^{k}\left[\prod_{i=1}^{n} \mu_{A_{i}^{k}}\left(x_{i}\right)\right]^{1-\gamma+\gamma / n}}{\sum_{k=1}^{m} \delta^{k}\left[\prod_{i=1}^{n} \mu_{A_{i}^{k}}\left(x_{i}\right)\right]^{1-\gamma+\gamma / n}}
$$

The cost function for minimizing the error is defined as:

$f\left(x^{p}\right)$ and $y^{p}$ are the output of the CFNN and the desired output for the $p$ th training datum, respectively. Then, we use a gradient descending method for minimizing the cost function to update the parameters for backpropagation. The new compensatory degree $\gamma(t+1)$ can be described below see Fig. (3):

$$
\begin{aligned}
& E^{P}=\frac{1}{2}\left[f\left(x^{p}\right)-y^{p}\right]^{2} \\
& c(t+1)=c(t)-\left.\eta\left\{\frac{2 c(t) d^{2}(t)}{\left[c^{2}(t)+d^{2}(t)\right]^{2}}\right\} \frac{\partial E^{p}}{\partial \gamma}\right|_{t} \\
& d(t+1)=d(t)+\left.\eta\left\{\frac{2 c(t) d^{2}(t)}{\left[c^{2}(t)+d^{2}(t)\right]^{2}}\right\} \frac{\partial E^{p}}{\partial \gamma}\right|_{t} \\
& \gamma(t+1)=\frac{c^{2}(t+1)}{c^{2}(t+1)+d^{2}(t+1)}
\end{aligned}
$$

Where $\eta$ is learning rate and $t=0,1,2, \mathrm{~L}$.

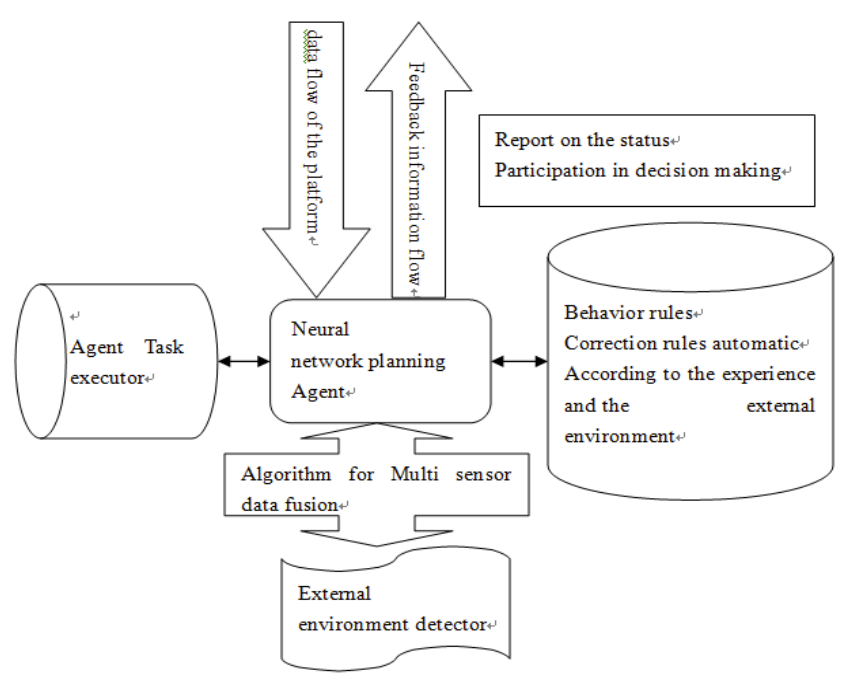

Fig. (3). The structure of individual agent members of the MAS. 
The algorithm designed by compensatory fuzzy neural network has powerful adaptive information processing. In this structure, function of neural network node and function of connection weights with fuzzy membership and the fuzzy control rules are well combined together. It is a good solution to determine the membership function of fuzzy reasoning and the learning ability problem of similar traditional algorithms. The compensatory fuzzy neural network does not need to establish accurate mathematics model, which has powerful self learning. It can be combined with the experience of the knowledge base, and accurately obtain flow characteristic through proper training can, and can make the correct trend prediction. It has simple structure, adaptability and robustness; good to meet the agent members automatically rule revision demand.

\section{CONCLUSION}

In this paper, Comprehensive Information Platform designed for collaborative manufacturing was presented with an application case in garment industry. In addition, technical details about business resources planning model in manufacturing system, especially, the relation of subsystems are expressed clearly by figures, and then the design of the optimal production planning algorithm to keep the manufacture financing stability in product cycle is discussed in the following sections. We mainly concern and research into Mass-, AS parallel decision in integrated infrastructure for agile enterprise, including the analysis of Information Exchange Rules for MAS Members, Individual Behavior Rules and Its correction by environment driven, MAS decision rules for members in the algorithm, the solution to the problem \& algorithm design in The decision-making operation burden for IIAE. All of them are elaborated in turn. More elaborating performance report will come up after long period of observational and practical use. All technical details touched upon run in application, and the main improvement in its performance can only come with application.

\section{CONFLICT OF INTEREST}

The authors confirm that this article content has no conflict of interest.

\section{ACKNOWLEDGEMENTS}

Declared none.

\section{REFERENCES}

[1] N. G. Shaw, A. Mian, and S. B. Yadav, "A comprehensive agentbased architecture for intelligent information retrieval in a distrib- uted heterogeneous environment," Decision Support Systems, vol. 32, pp. 401- 415, 2002.

[2] N. R. Jennings, K. Sycara, and M. Wooldridge, "A Roadmap of Agent Research and Development," Autonomous Agents and MultiAgent Systems, vol. 1, pp. 275-306, 1998.

[3] M. Wooldridge, and N.R. Jennings, "Intelligent agents: theory and practise," Knowledge Engineering Review, vol. 10, no. 2, pp. 115$152,1995$.

[4] M. Wooldridge, "Semantic Issues in the Verification of Agent Communication Languages," Autonomous Agents and Multi-Agent Systems, vol. 1, pp. 1387-2532, 2000.

[5] N. Vulkan, and N. R. Jennings, "Efficient mechanisms for supply of services in multi-agent environment," Decision Support Systems, vol. 28, pp. 5-19, 2000.

[6] O.B. Kwon, and K.C. Lee, "MACE: multi-agent coordination engine to resolve conflicts among functional units in an enterprise," Expert Systems with Applications, vol. 23, pp. 376-389, 2002.

[7] Gong-Baojun, "Mutl-Agent based enterprise information integration theory and Its Application Research," Doctoral Dissertation of Zhejiang University, vol. 6, 2001.

[8] A. Bergman, and M. Tennenholtz, "On the Natural Selection of Market Choice," Autonomous Agents and Multi-Agent Systems, vol. 4, pp. 1387-2532, 2002.

[9] S. Parsons, and M. Wooldridge, "Game Theory and Decision Theory in Multi-Agent Systems," Autonomous Agents and Multi-Agent Systems, vol. 3, pp. 1387-2532, 2002.

[10] J. Grant, S. Kraus, and D. Perlis, "A Logic for Characterizing Multiple Bounded Agents," Autonomous Agents and Multi-Agent Systems, vol. 4, pp. 1387-2532, 2000.

[11] M. V. N. Prasad, and V. R. Lesser, "Learning Situation-Specific Coordination in Cooperative Multi-agent Systems," Autonomous Agents and Multi-Agent Systems, vol. 2, pp. 1387-2532, 1999.

[12] N. R. Jennings, "Cooperation in Industrial Multi-Agent System," In: Word Scientific Publishing Co., Ltd. New York. 1994.

[13] D. Y. Liu, and J. H. Chen, "One-one bargaining negotiation process in Multi-agent system," In: Proc. of CSCW in design, pp. 150-159, 1996.

[14] W. Shi, L. Zhang, and Y. Jin, "Integrating Communicative Action and Confliction Management among Autonomous Agents," In: Proc. of '02 International Conference on Control and Automation, Xiamen, China, 2002.

[15] S. Mathiyalakan, "A methodology for controlled empirical investigation of membership continuity and change in GDSS groups," Decision Support Systems, vol. 32, pp. 279- 295, 2002.

[16] H. Wang, S. Liao, and L. Liao, "Modeling constraint-based negotiating agents," Decision Support Systems, vol. 33, pp. 201-217, 2002.

[17] M.P. Greg, and R. Jennings, "Foundations of Distributed Artificial Intelligence," New York: John Wiley \& Sons, Inc., 1996.

[18] S. Kraus, "Negotiation and cooperation in multi-agent environments," Artificial Intelligence, vol. 94, pp. 79-97, 1997.

[19] N. Karacapilidis, "Integrating new information and communication technologies in a group decision support system," International Transactions in Operations Research, vol. 7, pp. 487-507, 2000.

[20] J. Rees, and R. Barkhi, "The problem of highly constrained tasks in group decision support systems," European Journal of Operational Research, vol. 135, pp. 220-229, 2001.

[21] J. H. Miller, C. T. Butts, and D. Rode, "Communication and cooperation," Journal of Economic Behavior \& Organization, vol. 47, pp. 179-195, 2002.

\footnotetext{
Received: May 26, 2015

Revised: July 14, 2015

Accepted: August 10,2015

(C) J.L. Su; Licensee Bentham Open.

This is an open access article licensed under the terms of the (https://creativecommons.org/licenses/by/4.0/legalcode), which permits unrestricted, noncommercial use, distribution and reproduction in any medium, provided the work is properly cited.
} 